\title{
On the structure of the supplementary series of unitary irreducible representations of the proper, ortochronous Lorentz group
}

\author{
A.Staruszkiewicz \\ Institute of Physics, Jagellonian University, \\ Reymonta 4, 30-059 Kraków, Poland
}

Received January 26, 1998

Representations from the supplementary series of unitary irreducible representations of the proper, ortochronous Lorentz group are labelled by the parameter $z, 0<z<1$. There are qualitative differences between representations with $0<z<1 / 2$ and those with $1 / 2<z<1$. Two such differences are described in this paper: the probability density of parabolic rotations in a spherically symmetric state is singular at the origin for $0<z<1 / 2$ but regular for $1 / 2<z<1$; the Casimir operator of the little group, which preserves a space-like vector, has for $0<z<1 / 2$ a bound state which disappears for $1 / 2<z<1$.

Key words: Lorentz group, unitary representations

PACS: $02.20 . Q$

\section{Introduction and motivation}

This paper is dedicated to Professor Roman Gaida on the occasion of his $70^{\text {th }}$ birthday. Professor Gaida has always been interested in problems in which both the theory of relativity and quantum mechanics play a role. Self-consistent union of these two great theories remains an unsolved problem of modern theoretical physics. Nothing illustrates better the immense difficulty of this problem than the phenomenon of charge quantization: electric charges of all elementary particles seem to be exactly the same. In the case of electron and proton, their charges are of equal magnitude with the experimental accuracy $1: 10^{-21}$. Coincidence of two independent quantities, which holds with such a fantastic accuracy, obviously calls for an explanation.

My view on the problem of charge universality can be summarized as follows. It is completely absurd to suppose that a coincidence which holds with the accuracy $1: 10^{-21}$ is of a dynamical origin. There must be a purely kinematical 
principle which explains this coincidence. I formulated this principle in [1]. The quantum mechanics of the electric charge described in this paper allows us to treat the Coulomb field quantum-mechanically i.e. as a ray in a Hilbert space. In [2] I proved the following theorem: the quantum Coulomb field, when decomposed into unitary irreducible representations of the proper, ortochronous Lorentz group, contains only the main series if $e^{2} / \hbar c>\pi . e^{2} / \hbar c$ is the fine structure constant in unrationalized Gaussian units. If, however, $0<e^{2} / \hbar c<\pi$, then the quantum Coulomb field contains the main series and a single representation from the supplementary series corresponding to the special value of the Casimir operator

$$
C_{1}=-\frac{1}{2} M_{\mu \nu} M^{\mu \nu}=\frac{e^{2}}{\pi \hbar c}\left(2-\frac{e^{2}}{\pi \hbar c}\right) .
$$

This theorem seems to be of fundamental importance because it establishes a functional relation between the fine structure constant and a purely kinematical quantity, namely the parameter which labels unitary irreducible representations from the supplementary series. It is thus natural to investigate how various properties of the supplementary series depend on the numerical value of the parameter $z$, which selects a single representation from the series. Two results of such an investigation are reported in this paper.

\section{The supplementary series}

The supplementary series of unitary irreducible representations of the proper, ortochronous Lorentz group was discovered independently by Gelfand and Neumark [3], Bargmann [4] and Harish-Chandra [5]. It is described in the book by Gelfand, Graev and Vilenkin [6]. Let me summarize very briefly the relevant terminology and notation.

The four-vector $k$ is called null if $k k=\left(k^{0}\right)^{2}-\left(k^{1}\right)^{2}-\left(k^{2}\right)^{2}-\left(k^{3}\right)^{2}=0$. It is called future oriented if $k^{0}>0$. The set of all future oriented null vectors forms the future light cone. The light cone consists of rays. A ray is a set of null vectors parallel to a given null vector. The set of all rays has the Lorentz invariant volume two-form, which Gelfand, Graev and Vilenkin give on page 426 of their book [6]:

$$
\mathrm{d}^{2} k=\frac{k^{1}\left[\mathrm{~d} k^{2} \mathrm{~d} k^{3}\right]+k^{2}\left[\mathrm{~d} k^{3} \mathrm{~d} k^{1}\right]+k^{3}\left[\mathrm{~d} k^{1} \mathrm{~d} k^{2}\right]}{k^{0}} .
$$

The function $f(k)$ defined on the future light cone is said to be homogeneous of degree $z-2,0<z<1$, if

$$
f(\lambda k)=\lambda^{z-2} f(k) \text { for each } \lambda>0 .
$$

Functions homogeneous of degree $z-2$ form a linear space. Introducing in this space the Lorentz invariant and the positive definite scalar product

$$
\langle f \mid g\rangle=\int \frac{\mathrm{d}^{2} k \mathrm{~d}^{2} l}{(k l)^{z}} \overline{f(k)} g(l)
$$

we obtain a Hilbert space in which the proper, ortochronous Lorentz transformations are unitary and irreducible. 


\section{Probability density of parabolic rotations in a spherically symmetric state from the supplementary series}

There are three kinds of one-parameter subgroups of the proper, ortochronous Lorentz group: rotations which preserve a time-like plane, hyperbolic rotations, sometimes called "boosts", which preserve a space-like plane and parabolic rotations preserving a null plane. If $M_{\mu \nu}$ are generators of the proper, ortochronous Lorentz group, then, for example, $M_{12}$ generates a rotation, $M_{03}$ generates a hyperbolic rotation, while $M_{01}+M_{12}$ generates a parabolic rotation.

Let $|f\rangle,\langle f \mid f\rangle=1$ be an arbitrary state and $A$ - a self-adjoint operator i.e. observable in Dirac's terminology. The matrix element

$$
\left\langle f\left|\mathrm{e}^{i \lambda A}\right| f\right\rangle
$$

where $\lambda$ is a real number, is called an autocorrelation function. Its Fourier transform

$$
p_{f}(\mu)=\frac{1}{2 \pi} \int_{-\infty}^{+\infty} \mathrm{d} \lambda \mathrm{e}^{-i \mu \lambda}\left\langle f\left|\mathrm{e}^{i \lambda A}\right| f\right\rangle
$$

is a probability density of the observable $A$ in the state $|f\rangle$. By the well-known theorem of functional analysis this Fourier transform is non-negative and summable to 1 , thus, it is indeed a probability density.

I shall calculate the probability density of parabolic rotations in the spherically symmetric state $|u\rangle$ from the supplementary series. $u$ is a four-velocity of the inertial reference frame in which the state $|u\rangle$ is spherically symmetric. It is easy to calculate that

$$
\langle u \mid v\rangle=\frac{\sinh [(1-z) \sigma]}{(1-z) \sinh \sigma}
$$

where $\sigma$ is a hyperbolic angle between the four-velocities $u$ and $v$. However, this angle is not equal to the canonical parameter $\lambda$ of the parabolic rotation which transforms $u$ into $v$. One rather has

$$
1+\frac{1}{2} \lambda^{2}=\cosh \sigma
$$

or

$$
\lambda=2 \sinh \frac{\sigma}{2} .
$$

Hence, putting $x=\sigma / 2$, we have

$$
p(\mu)=\frac{1}{2 \pi} \int_{-\infty}^{+\infty} \mathrm{d} x \cos (2 \mu \sinh x) \frac{\sinh [2(1-z) x]}{(1-z) \sinh x} .
$$

This integral can be calculated in terms of Bessel functions:

$$
p(\mu)=\frac{\sin [\pi(1-z)]}{\pi(1-z)} \int_{2|\mu|}^{\infty} \mathrm{d} x K_{2(1-z)}(x),
$$


where

$$
K_{\nu}(x)=\int_{0}^{\infty} \mathrm{d} t \mathrm{e}^{-x \cosh t} \cosh (\nu t) .
$$

It is seen that for $\mu \rightarrow 0$

$$
\lim _{\mu \rightarrow 0} p(\mu)
$$

exists for $1 / 2<z<1$ but does not exist for $0<z<1 / 2$. This shows that there is an observable, qualitative difference between representations with $0<z<1 / 2$ and those with $1 / 2<z<1$.

\section{Spectrum of the Casimir operator of a little group of a space- like vector}

A set of proper, ortochronous Lorentz transformations which preserve a fixed vector $a$ is called a little group of this vector. If vector $a$ is time-like, then its little group is simply a group of rotations in the inertial reference frame which moves with the four-velocity $a$. In what follows I consider the little group of the space-like vector $a, a a=-1$.

Consider the function

$$
f(k)=\delta(a k) f_{1}(k),
$$

where $\delta$ is the Dirac delta function, while $f_{1}(k)$ is a function homogeneous of degree $z-1$,

$$
f_{1}(\lambda k)=\lambda^{z-1} f_{1}(k) \text { for each } \lambda>0 .
$$

Assume that $f_{1}(k)$ is well behaved, e.g. continuous at each $k$ in the circle $a k=0$. Then, the state $f(k)$ is normalizable for $0<z<1 / 2$ :

$$
\langle f \mid f\rangle=\int \frac{\mathrm{d}^{2} k \mathrm{~d}^{2} l}{(k l)^{z}} \delta(a k) \overline{f_{1}(k)} \delta(a l) f_{1}(l)<\infty
$$

for $0<z<1 / 2$. Take $a$ parallel to the third axis, $a k=k^{3}$. Introduce the spherical coordinates

$$
\begin{aligned}
& k^{1}=k^{0} \sin \vartheta \cos \varphi, \\
& k^{2}=k^{0} \sin \vartheta \sin \varphi, \\
& k^{3}=k^{0} \cos \vartheta,
\end{aligned}
$$

and write $f(k)$ in the form:

$$
\begin{aligned}
f(k) & =\delta\left(k^{3}\right) f_{1}(k) \\
& =\delta\left(k^{3}\right)\left(k^{0}\right)^{z-1} f_{2}\left(\frac{k^{1}+i k^{2}}{k^{0}+k^{3}}\right) \\
& =\delta\left(k^{3}\right)\left(k^{0}\right)^{z-1} f_{2}\left(\mathrm{e}^{i \varphi}\right) .
\end{aligned}
$$


Then,

$$
\langle f \mid f\rangle=2^{-z} \int_{0}^{2 \pi} \mathrm{d} \varphi \int_{0}^{2 \pi} \mathrm{d} \psi \frac{\overline{f_{2}\left(\mathrm{e}^{i \varphi}\right)} f_{2}\left(\mathrm{e}^{i \psi}\right)}{\left|\sin \frac{\varphi-\psi}{2}\right|^{2 z}}
$$

$0<z<1 / 2$.

This is the scalar product given by Bargmann [4] on page 617 of his paper. The Casimir operator of the little group of vector $a$ has the value $z(1-z)<1 / 4$,

$$
\left(M_{01}^{2}+M_{02}^{2}-M_{12}^{2}\right) f=z(1-z) f .
$$

Thus, we have a normalizable eigenstate of the operator $M_{01}^{2}+M_{02}^{2}-M_{12}^{2}$, i.e. a bound state. No such a bound state exists for $1 / 2<z<1$, which means that representations with $0<z<1 / 2$ are qualitatively different from those with $1 / 2<z<1$.

\section{Acknowledgements}

This work was supported in part by the KBN grant 2 P03B 09008.

\section{References}

1. A. Staruszkiewicz, Ann. Phys. (N.Y.) 190 (1989) 354.

2. A. Staruszkiewicz, Acta Phys. Pol. B23 (1992) 591. This paper is marred by several misprints, which are there because the journal did not take into account my proofs. For a corrected version see ERRATUM, Acta Phys. Pol. B23 (1992) 959.

3. I.M. Gelfand and M.A. Neumark, Izv. Akad. Nauk SSSR (mathematical series) 11 (1947) 411.

4. V. Bargmann, Annals of Mathematics 48 (1947) 568.

5. Harish-Chandra, Proc. Roy. Soc. London, Ser. A 189 (1947) 372.

6. I.M. Gelfand, M.I. Graev, and N. Ya. Vilenkin, Generalized Functions, Vol. V, Moscow 1962. 


\title{
Про структуру додаткової серії унітарних незвідних зображень власної ортохронної групи Лоренца
}

\author{
А.Старушкевич \\ Яґеллонський університет, Інститут фізики, \\ вул. Реймонда, 4, Краків, 30-059, Польща \\ Отримано 26 січня 1998 р.
}

Зображення з додаткової серії унітарних незвідних зображень власної ортохронної групи Лоренца характеризуються параметром $z$, $0<z<1$. Існують якісні відмінності між зображеннями із $0<z<1 / 2$ та із $1 / 2<z<1$. Дві такі відмінності описано у статті: густина ймовірности параболічних поворотів у сферично-симетричному стані синґулярна в початку координат для $0<z<1 / 2$, але реґулярна для $1 / 2<z<1$; оператор Казимира малої групи, яка зберігає просторово-подібний вектор, має для $0<z<1 / 2$ зв'язаний стан, який зникає, коли $1 / 2<z<1$.

Ключові слова: група Лоренца, унітарні зображення

PACS: $02.20 . Q$ 